\title{
Interdependence of kallikrein-related peptidases in proteolytic networks
}

\author{
Nathalie Beaufort ${ }^{1}$, Karolina Plaza ${ }^{1,2}$, Daniel \\ Utzschneider $^{1}$, Amelie Schwarz ${ }^{1}$, Julia M. Burkhart ${ }^{1}$, \\ Sabine Creutzburg ${ }^{1}$, Mekdes Debela ${ }^{1,3, a}$, Manfred \\ Schmitt $^{1}$, Christian Ries ${ }^{4}$ and Viktor Magdolen ${ }^{1} *$ \\ ${ }^{1}$ Department of Obstetrics and Gynecology, Technical \\ University of Munich, Ismaninger Str. 22, D-81675 \\ Munich, Germany \\ ${ }^{2}$ Department of Microbiology, Faculty of Biochemistry, \\ Biophysics and Biotechnology, Jagiellonian University, \\ ul. Gronostajowa 7, 30-387 Krakow, Poland \\ ${ }^{3}$ Proteinase Research Group, Max Planck Institute of \\ Biochemistry, Am Klopferspitz 18, D-82152 Martinsried, \\ Germany \\ ${ }^{4}$ Division of Clinical Chemistry and Clinical Biochemistry, \\ Ludwig Maximilians University of Munich, Nussbaumstr. \\ 20, D-80336 Munich, Germany \\ * Corresponding author \\ e-mail: viktor.magdolen@ lrz.tum.de
}

\begin{abstract}
Human kallikrein-related peptidases (KLKs) are 15 homologous serine proteases involved in several (patho)physiological processes, including cancer. Secreted as precursors, they are activated upon proteolytic release of a short propeptide. We searched for interconnection of KLKs within extracellular proteolytic networks leading to activation of protease zymogens and found that (i) pro-KLK activation by other KLKs is scarce, with the exception of pro-KLK11, which is efficiently activated by KLK4 and 5; (ii) pro-KLK4 is activated by matrix metalloproteinase 3 ; and (iii) trypsinlike KLKs efficiently activate the serine protease urokinase. Our observations provide new insights into the regulation of these important tumor-associated proteases.
\end{abstract}

Keywords: cancer; matrix metalloproteinases; proteases; urokinase; zymogen activation.

\section{Introduction}

Proteolysis is one of the essential and widespread post-translational processes affecting proteins in all living organisms (Barrett, 2004; Lopez-Otin and Bond, 2008). Proteases not only degrade other proteins but also positively regulate,

\footnotetext{
a Present address: Medical Research Council, Laboratory of Molecular Biology, Structural Division, G8, Hills Road, Cambridge CB2 OQH, UK.
}

directly or indirectly, the activity of numerous bioactive molecules and are consequently involved in an impressive number of (patho)physiological processes (Lopez-Otin and Bond, 2008). Remarkable examples are provided by human kallikrein-related peptidases (KLKs), which recently came into focus because of their clinical relevance as diagnostic, prognostic and/or predictive tumor biomarkers, and as potential therapeutic targets (Borgono and Diamandis, 2004).

The KLK superfamily includes 15 serine (Ser-) proteases (KLK1-15) whereby all genes are located on chromosome 19 q13.4 and constitute the largest protease gene cluster in the human genome (Borgono and Diamandis, 2004). These 25-30 kDa secreted enzymes display a wide expression pattern in tissues and body fluids (Shaw and Diamandis, 2007) and participate in several physiological processes such as skin desquamation, tooth development, neuronal plasticity or semen liquefaction (Borgono and Diamandis, 2004; Pampalakis and Sotiropoulou, 2007). Along with this, some KLKs are aberrantly expressed in pathologies, including skin, neurodegenerative and/or malignant disorders, e.g., prostate, breast and ovarian cancer (Borgono and Diamandis, 2004; Pampalakis and Sotiropoulou, 2007).

KLKs are secreted as zymogens and are extracellularly activated via proteolytic removal of a rather short $\mathrm{N}$-terminal propeptide (Borgono and Diamandis, 2004). This process thus represents a key step in the regulation of their activity. Upon activation, they display a trypsin- or chymotrypsin-like enzymatic activity (Borgono and Diamandis, 2004; Debela et al., 2006b, 2008). As KLKs are devoid of any other domain besides the protease domain, their function is likely to rely exclusively on their proteolytic activity towards their substrates including extracellular matrix proteins, growth factors and membrane receptors (Borgono and Diamandis, 2004; Debela et al., 2008; Hollenberg et al., 2008; Emami and Diamandis, 2010). Consequently, KLK activation has been actively investigated in the past 5 years and is currently proposed to occur via reaction cascades during which one given KLK activates another KLK (Borgono and Diamandis, 2004; Pampalakis and Sotiropoulou, 2007; Yoon et al., 2007, 2009; Debela et al., 2008).

In this context, we designed a strategy allowing highthroughput analysis of putative pro-KLK activators, which we searched among tumor-related Ser-proteases such as KLKs themselves, as well as urokinase (uPA) (Dano et al., 2005). In parallel, we tested KLKs for their capacity to affect the pro-uPA zymogen. Furthermore, in the particular case of pro-KLK4, which displays a Gln residue at the P1 position of its activation cleavage site, whereas KLK zymogens rather display an Arg or a Lys residue (Borgono and Diamandis, 2004), we challenged pro-KLK4 with some tumor-associated 
matrix metalloproteinases (MMP-2, -3, -9, and, membrane type 1-/MT1-MMP) (Itoh and Nagase, 2002; Freije et al., 2003).

\section{Results}

\section{Activation and inactivation of pro-KLKs by KLKs}

To allow for efficient, high-throughput screening, pro-KLKs were cloned and expressed in Escherichia coli as chimeric proteins bearing $\mathrm{N}$ - and C-terminal extensions (i.e., a Histag and a Tag100 epitope, respectively, Figure 1A). Upon exposure of pro-KLKs to potential activators, we analyzed the reaction mixture by immunoblot, considering that activation would result in a loss of His-tag reactivity, along with the occurrence of a Tag100-reactive species displaying a slightly lower molecular weight (Figure 1A). Using this strategy, we were able to investigate 63 pro-KLK/KLK couples. Overall results are summarized in Table 1, while selected examples are depicted in Figure 1B.

Pro-KLK activation by other KLKs was limited to proKLK11, which we found to be efficiently processed from an approximate $27 \mathrm{kDa}$ species, reacting both with the His-tag and the Tag $100 \mathrm{mAb}$, into an approximate $23 \mathrm{kDa}$, His-tag deficient form, by KLK4 and KLK5 (Figure 1B, enzyme substrate molar ratio $1 / 10,5 \mathrm{~h}$ exposure), and to a lesser extent by KLK6 and KLK8 (Figure 1B, note the persistence of the His-tagged, uncleaved form). Amino-terminal microsequencing indicated that the KLK11 species generated upon exposure to KLK4 or KLK5, actually bear the Ile-Ile-LysGly amino-terminus of mature KLK11 and, thus, correspond to the active form of the protease.

By contrast, (pro-)KLKs appeared remarkably prone to proteolytic inactivation or degradation (Table 1). For instance, pro-KLK11 activation, particularly by KLK5, coincides with the occurrence of an approximate $15 \mathrm{kDa}$, Tag100-reactive degradation product (Figure 1A and B). As this species is not observed when pro-KLK11 is exposed to KLK11 (Figure 1B), it rather results from direct processing of (pro-)KLK11 by other KLKs, than from KLK11 autodegradation. Another relevant example is provided by proKLK13 (approximately $25 \mathrm{kDa}$ ). Indeed, whereas the non-treated zymogen remains stable upon incubation at $37^{\circ} \mathrm{C}$, it is converted by $\mathrm{KLK} 4$ into an approximate $21 \mathrm{kDa}$, His-tag-reactive, Tag100-deficient form, indicating, again, proteolytic inactivation of the enzyme (Figure 1A, B). Finally, upon incubation with KLK5, pro-KLK5 looses both the His-tag and the Tag100 reactivity, reflecting its extensive cleavage/degradation (Figure 1A, B), as also confirmed by silver staining (data not illustrated).

\section{Interplay of KLKs with (pro-)urokinase}

In addition to KLKs, uPA is another well-characterized, tumor-associated, trypsin-like Ser-protease. Indeed, this enzyme has been known for a long time to contribute to tumor cell growth and dissemination (Sperl et al., 2001; Dano et al., 2005). As crosstalk is a common feature for human proteolytic systems, we investigated the capacity of uPA to target pro-KLKs, as well as the reciprocal activity of KLKs to affect pro-uPA.

Upon exposure to uPA, none of the pro-KLKs was found to be processed (data not shown). In contrast, as previously reported for KLK2 and KLK4 (Frenette et al., 1997; Takayama et al., 1997, 2001; Beaufort et al., 2006), all tested KLKs, with the exception of KLK7 (i.e., the unique chymotrypsinlike peptidase among the enzymes which we investigated), converted single chain pro-uPA (approximately $47 \mathrm{kDa}$ ) into two molecular species (Figure 2A, upper panel) that seem similar to those generated upon exposure to its canonical activator plasmin: the approximate $29 \mathrm{kDa}$ mature catalytic domain (B-chain), and the approximate $17 \mathrm{kDa}$ binding domain (A-chain). Accordingly, proteolytic activity measurement demonstrated that pro-uPA is indeed activated by KLK4, 5, 6, 8, 10 and 11, whereas KLK7 leaves the zymogen inactive (Figure 2A, lower panel and Figure 2B, for KLK5, 6 and 7). In some cases, a further degradation of the uPA A-chain was also observed.

\section{Activation of pro-KLK4 by matrix metalloproteinases}

Differing from other KLKs, pro-KLK4 displays a Gln residue at the P1 position of its activation cleavage site (Borgono and Diamandis, 2004) and is thus rather unlikely to be activated by trypsin- or chymotrypsin-like enzymes. Apart from Ser-proteases, several soluble or membrane-associated MMPs are also expressed in the extracellular microenvironment of malignant tumor cells and contribute to tumorigenesis and metastatic spread (Itoh and Nagase, 2002; Freije et al., 2003). Among these proteases, we evaluated the capacity of MMP-2, -3, -9, and MT1-MMP to affect pro-KLK4. Additionally, a recently reported variant of MMP-9, MMP-9 $\Delta$, was also investigated (Ries et al., 2007). As illustrated in Figure 3A, MMP-3 converted recombinant pro-KLK4 into a slightly lower molecular mass species. This processing, which was detectable after $4 \mathrm{~h}$ exposure to MMP-3 in an enzyme/substrate molar ratio of $1 / 10$ and increased over time, actually corresponds to activation of the KLK4 zymogen, as indicated by proteolytic activity measurement (Figure 3B). In contrast, MMP-2, $-9,-9 \Delta$ and MT1-MMP, neither activated nor degraded pro-KLK4 (data not shown).

\section{Discussion}

Although their exact (patho)physiological functions remain to be clarified, KLKs, including the well-known prostate specific antigen (PSA/KLK3), have recently been the focus of attention as promising tumor biomarkers (Borgono and Diamandis, 2004). Particularly, identification of the proteolytic activators of these enzymes, which are currently thought to comprise Ser-proteases, including KLKs themselves, has emerged as a challenging task (Borgono and Diamandis, 2004; Pampalakis and Sotiropoulou, 2007). However, analysis of pro-KLKs activation is rather difficult owing to particular (pro-)KLK features. For instance, with the exception of pro-KLK5, all pro-KLKs carry a short pro-domain (i.e., 


\section{A}
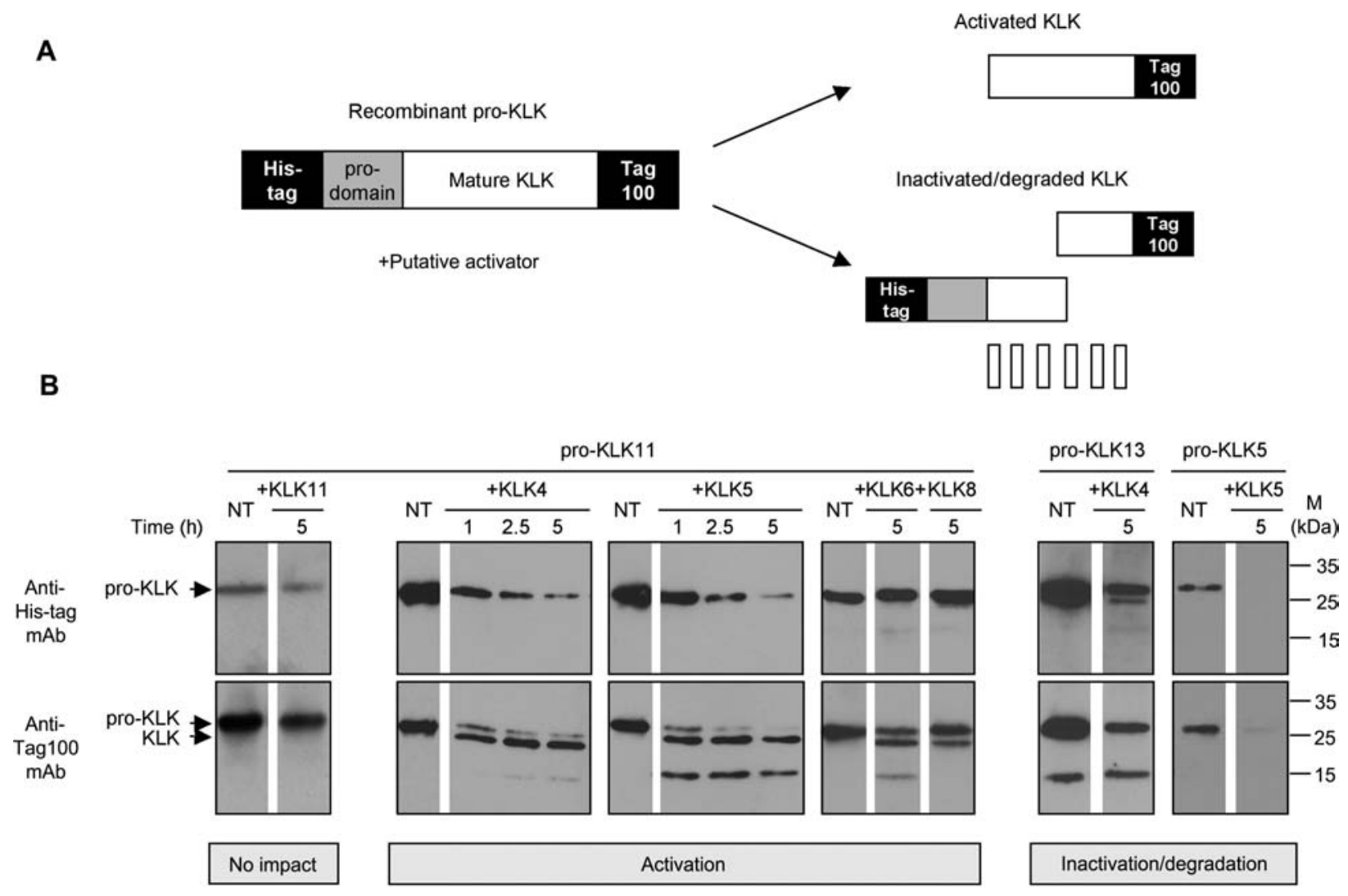

Figure 1 Activation and inactivation/degradation of human pro-KLKs by KLKs.

(A) Recombinant pro-KLKs were generated as chimeric proteins bearing an amino-terminal His-tag and a carboxy-terminal Tag 100 epitope. Such constructs allow efficient discrimination between activation and proteolytic inactivation/degradation of the zymogens. (B) Pro-KLKs were left untreated as control (NT), or exposed to KLKs in an enzyme/substrate molar ratio of $1: 10$, for 1 to $5 \mathrm{~h}$ at $37^{\circ} \mathrm{C}$, before the reaction mixture was analyzed by immunoblotting, using an anti-His-tag mAb (upper panel), and then an anti-Tag100 mAb (lower panel). Portions of the films corresponding to the location of relevant antigens and representative of at least two independent experiments are depicted. The position and molecular mass of standard proteins are indicated on the right-hand side.

4-15 amino acids). Removal of the pro-peptide is thus barely detectable via general protein electrophoresis procedures (Borgono and Diamandis, 2004). In addition, KLKs are highly homologous enzymes (Borgono and Diamandis, 2004), which do not only display similar molecular masses but also share macromolecular and synthetic substrates, as well as inhibitors. In several cases, it is therefore difficult to discriminate between an activated KLK and a KLK-type activator. Furthermore, it should be noted that commercial Abs are not available for all KLKs and that some display significant reactivity for more than one KLK (unpublished results). Our strategy, i.e., to analyze the processing of double-tagged pro-

Table 1 Processing of human pro-KLKs by KLKs.

\begin{tabular}{lccccccccc}
\hline & pro-KLK4 & pro-KLK5 & pro-KLK6 & pro-KLK7 & pro-KLK8 & pro-KLK10 & pro-KLK11 & pro-KLK13 & pro-KLK15 \\
\hline KLK4 & $\mathrm{D}$ & $\mathrm{D}$ & $\mathrm{D}$ & $\mathrm{N}$ & $\mathrm{D}$ & $\mathrm{D}$ & $\mathrm{A}$ & $\mathrm{D}$ & $\mathrm{D}$ \\
KLK5 & $\mathrm{D}$ & $\mathrm{D}$ & $\mathrm{D}$ & $\mathrm{D}$ & $\mathrm{D}$ & $\mathrm{N}$ & $\mathrm{A}$ & $\mathrm{N}$ & $\mathrm{D}$ \\
KLK6 & $\mathrm{N}$ & $\mathrm{N}$ & $\mathrm{N}$ & $\mathrm{N}$ & $\mathrm{N}$ & $\mathrm{N}$ & $\mathrm{A}$ & $\mathrm{N}$ & $\mathrm{N}$ \\
KLK7 & $\mathrm{D}$ & $\mathrm{D}$ & $\mathrm{D}$ & $\mathrm{N}$ & $\mathrm{D}$ & $\mathrm{D}$ & $\mathrm{N}$ & $\mathrm{N}$ & $\mathrm{D}$ \\
KLK8 & $\mathrm{N}$ & $\mathrm{D}$ & $\mathrm{N}$ & $\mathrm{N}$ & $\mathrm{D}$ & $\mathrm{N}$ & $\mathrm{A}$ & $\mathrm{N}$ & $\mathrm{N}$ \\
KLK10 & $\mathrm{N}$ & $\mathrm{N}$ & $\mathrm{N}$ & $\mathrm{N}$ & $\mathrm{D}$ & $\mathrm{N}$ & $\mathrm{N}$ & $\mathrm{N}$ & $\mathrm{N}$ \\
KLK11 & $\mathrm{D}$ & $\mathrm{D}$ & $\mathrm{N}$ & $\mathrm{D}$ & $\mathrm{N}$ & $\mathrm{N}$ & $\mathrm{N}$ & $\mathrm{N}$ & $\mathrm{N}$ \\
\hline
\end{tabular}

Upon exposure of pro-KLKs to KLKs, activation or inactivation/degradation were evaluated by immunoblotting, as depicted in the legend to Figure 1. (A: activation; D: inactivation/degradation; N: no impact). 


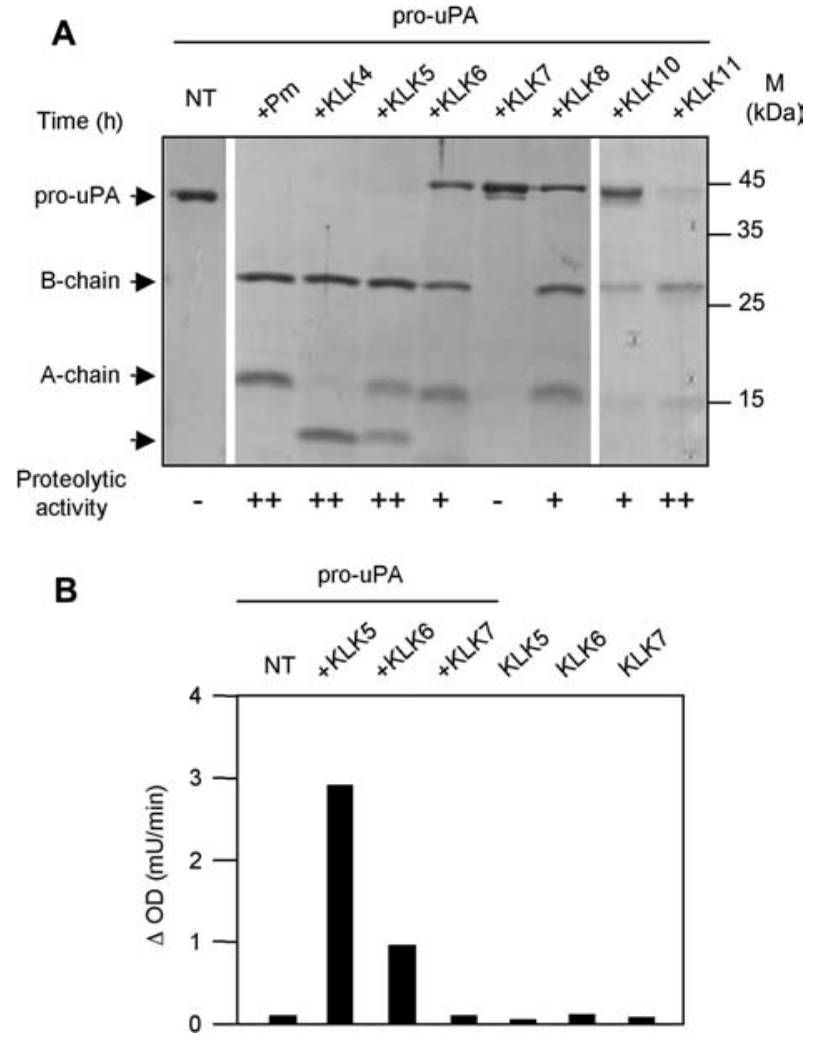

Figure 2 Activation of human pro-uPA by KLKs.

Pro-uPA was left untreated as a control (NT), exposed to its known activator plasmin (Pm), or to KLKs, in an enzyme/substrate molar ratio of $1: 10$, for $5 \mathrm{~h}$ at $37^{\circ} \mathrm{C}$. The reaction mixture was subsequently analyzed by protein electrophoresis followed by Coomassie Blue staining (A, upper panel), and by proteolytic activity measurement, using the uPA-selective chromogenic substrate Pefachrome uPA (A, lower panel and B).,+++ and - respectively depict high, moderate and no hydrolysis of the chromogenic substrate. One out of three analyses showing similar results is illustrated.

KLKs, circumvented these technical limits and allowed us to carry out high-throughput in vitro analysis of 77 pro-KLK/ potential activator couples. The $\mathrm{N}$ - and the C-termini of proenzyme forms of trypsin-like proteases are generally pointing out the core protease molecule (Gomis-Rüth et al., 2002). Thus, addition of tags is unlikely to affect the overall folding of the recombinant pro-enzyme forms.

In our study, pro-KLK cleavage leading to stable mature enzymes rarely occurred, as compared to the extensive activation cascades currently anticipated in the literature (Borgono and Diamandis, 2004; Pampalakis and Sotiropoulou, 2007). Indeed, Yoon et al. recently proposed that, with regard to 225 possible combinations, the so-called 'KLK activome' might consist of not less than 99 pro-KLK/KLK pairs (Yoon et al., 2007, 2009). Focusing on the (pro-)KLK subset which we analyzed, Yoon and coworkers described 20 positive reactions, whereas our procedure only indicated that KLK4, 5, 6 and 8 trigger pro-KLK11 activation. Concerning the efficacy of this processing, KLK4 and KLK5 appeared more potent than KLK6 and KLK8, a tendency which is in good agreement with the observations by Yoon and colleagues.
A
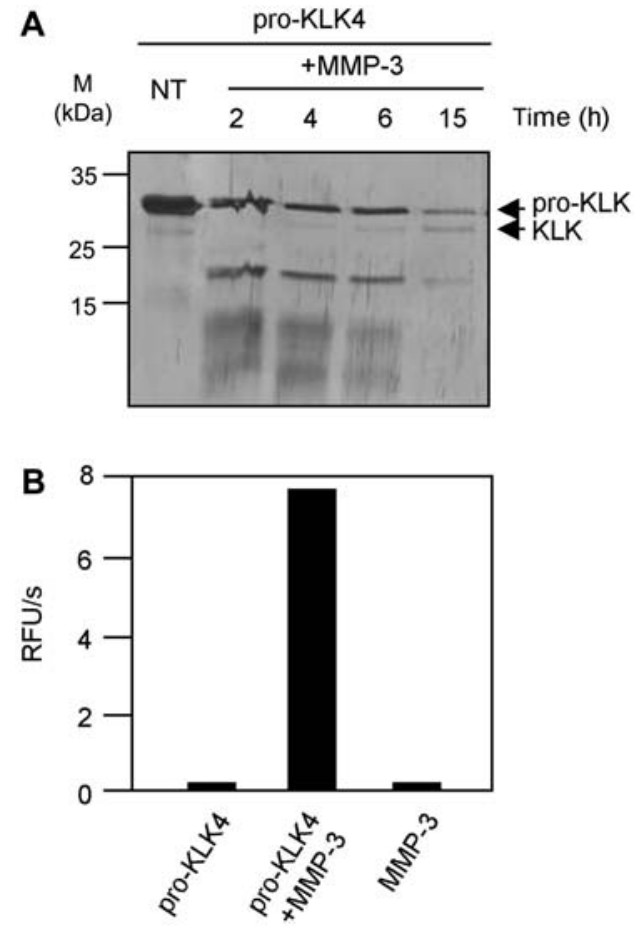

Figure 3 Activation of human pro-KLK4 by MMPs.

Pro-KLK4 was either left untreated as a control (NT) or exposed to MMP-3 in an enzyme/substrate molar ratio of $1: 10$, for $1,4,6$ or $15 \mathrm{~h}$ at $37^{\circ} \mathrm{C}$. (A) The reaction mixture was analyzed by electrophoresis followed by silver staining. (B) Pro-KLK4 was left untreated or exposed to MMP-3 for $15 \mathrm{~h}$ at $37^{\circ} \mathrm{C}$, before KLK4-related proteolytic activity was assayed using the fluorogenic substrate I-1120. Results illustrated are representative of two independent experiments (RFU: relative fluorescence unit).

Regarding the discrepancies, they probably result from significant differences between the two approaches. On the one hand, we acknowledge that a lower sensitivity of our Abbased detection system might explain some differences. On the other hand, Yoon and coworkers generated recombinant proteins encompassing an $\mathrm{N}$-terminal His-tag, followed by the pro-KLK peptide and the six amino-terminal residues of the KLK mature domain, fused to a soluble, protease-resistant, globular carrier protein (i.e., human fibroblast growth factor). This interesting model, however, omits the presence of putative protease-sensitive peptide bonds within the catalytic domain of enzymes, as it only monitors cleavage around the activation site. In fact, in our model, (pro-)KLKs appeared rather sensitive to proteolytic inactivation/degradation, whereas under similar experimental conditions, the KLK-activated serine protease domain of UPA was very stable. In vivo, glycosylation might stabilize (pro-)KLKs. Still, internal cleavage has been reported in vitro for several KLKs, either glycosylated or not (Borgono and Diamandis, 2004; Pampalakis and Sotiropoulou, 2007). Moreover, in the case of KLK2 and KLK3, the occurrence of truncated KLK species has actually been confirmed in vivo (Rittenhouse et al., 1998). Thus, we propose that proteolytic attack and inactivation of (pro-)KLKs might be a relevant regulation path- 
way, along with the sensitivity of these proteases to serpinor Kazal-type inhibitors, and to divalent ions (Borgono and Diamandis, 2004; Debela et al., 2006a, 2007).

In contrast to the well-known coagulation cascade that consists of sequential proteolytic activations, KLKs seem to be rather integrated within a proteolytic network which involves a large array of (pro-)proteases, with no clear hierarchy. To date, and apart from KLKs themselves, these proteases have been shown to include fibrinolytic enzymes (e.g., plasmin), coagulation proteases (e.g., thrombin), as well as the metalloproteases meprin $\alpha$ and $\beta$ (Borgono and Diamandis, 2004; Becker-Pauly et al., 2007; Pampalakis and Sotiropoulou, 2007; Yoon et al., 2007, 2008, 2009; Ohler et al., 2010). In the case of pro-meprin activation, up to now KLK4, 5 and 8 have been shown to activate the zymogen forms of meprin $\alpha$ and/or $\beta$ (Becker-Pauly et al., 2007; Ohler et al., 2010). We now report that trypsin-like KLKs are also potent activators of the urokinase zymogen, whereas they leave plasminogen inactive (unpublished data). Interestingly, plasmin as well as some MMPs are in turn able to trigger activation of some pro-KLKs. Particularly, as previously demonstrated for MMP-20 (Ryu et al., 2002), in the present paper, we report that MMP-3 triggers activation of proKLK4. This finding could be of pathophysiological relevance, as elevated levels of both MMP-3 and KLK4 were found to be associated with prostate cancer progression, where pro-KLK4 once activated by MMP-3 could influence growth and proliferation of tumor cells (Jung et al., 1997; Ramsay et al., 2008). Another metalloprotease, meprin $\beta$, was recently demonstrated to cleave pro-KLK7 two amino acids upstream its activation cleavage site (Ohler et al., 2010). Such processing, although leaving the zymogen inactive, actually primes pro-KLK7 toward further activation by endoproteases, but also, potentially, by exoproteases (Ohler et al., 2010). In line with this, dipeptidyl peptidase I was reported to activate pro-KLK4 (Tye et al., 2009).

In conclusion, although it deserves further confirmation in vivo, crosstalk between major human proteolytic systems certainly results in a rapid amplification of global proteolytic activity, as it is observed during, and thought to participate in, tumor cell migration and dissemination.

\section{Materials and methods}

\section{Reagents}

Recombinant human pro-uPA, expressed in E. coli, was a kind gift from A.G. Saunders (Grünenthal, Aachen, Germany), active KLK5 was generously provided by N. Schechter (University of Pennsylvania, Philadelphia, PA, USA). Human plasmin (4.6 U/mg), purified from plasma, was from Sigma-Aldrich (St. Louis, MO, USA). Active high-molecular weight (HMW-) uPA (rheothromb) was from DeltaSelect (Marburg, Germany). proMMP-9, proMMP-2 and the catalytic domain of MT1-MMP were purchased from Merck Biosciences/Calbiochem (Darmstadt, Germany). proMMP-9 $\Delta$ was isolated as described previously (Ries et al., 2007). The catalytic domain of MMP-3 was kindly provided by H. Nagase (Imperial College London, London, UK).

\section{Recombinant (pro-)KLK expression, purification and/or activation}

Recombinant active KLKs were expressed, purified and activated as reported elsewhere (Debela et al., 2006a,b, 2007). KLK4, 6, 7, 8, 10 and 11 are E. coli-expressed proteins, KLK5 was produced in insect cells.

Table 2 N-terminus of the analyzed recombinant KLK zymogen forms.

\begin{tabular}{llll}
\hline & \multicolumn{1}{c}{ N-terminal tag } & \multicolumn{1}{c}{ Pro-peptide } & $\begin{array}{c}\text { N-terminus of the } \\
\text { mature enzyme }\end{array}$ \\
\hline pro-KLK4 & MRGSHHHHHHGS & SCSQ $\downarrow$ & IINGEDC $\ldots$ \\
pro-KLK5 & MRGSHHHHHHGS & $\begin{array}{l}\text { NNDVSCDHPSNTVPSGSN } \\
\text { QDLGAGAGEDARSDDSSSR } \downarrow\end{array}$ & IINGSDC... \\
pro-KLK6 & MRGSHHHHHHGS & EEQNK $\downarrow$ & LVHGGPC... \\
pro-KLK7 & MRGSHHHHHHGS & EEAQGDK $\downarrow$ & IIDGAPC... \\
pro-KLK8 & MRGSHHHHHHGS & QEDK $\downarrow$ & VLGGHEC ... \\
pro-KLK10 & MRGSHHHHHHGS & ALLPQNDTR $\downarrow$ & LDPEAYGAPC... \\
pro-KLK11 & MRGSHHHHHHGS & GETR $\downarrow$ & IIKGFEC... \\
pro-KLK13 & MRGSHHHHHHGS & QESSKVLNTNGTSGF $\downarrow$ & LPGGYTC... \\
pro-KLK15 & MRGSHHHHHHGS & AQDGDK $\downarrow$ & LLEGDEC... \\
\hline
\end{tabular}

All recombinant pro-forms harbor an $\mathrm{N}$-terminal His-tag, followed by a short peptide consisting of 4-37 amino acids. The activation site is indicated by an arrow. All KLK proteases, with the exception of KLK4 and KLK13, are supposed to be activated by trypsin-like proteases by cleavage after an arginine or lysine residue. The distance of the N-terminus of mature human KLK proteases to the first highly conserved cysteine residue generally consists of six amino acids, with the exception of KLK10 which displays an unusual insertion of three additional amino acids. 
For recombinant pro-KLK production, the coding sequence of each natural pro-KLK (merops.sanger.ac.uk; see also Table 2) was inserted in frame with the sequences encoding an (His) $)_{6}$-tag and a Tag100 epitope, respectively, into the bacterial expression vector pQE-Double-Tag100 (Qiagen, Hilden, Germany). The resulting fusion gene encodes the individual pro-KLK framed by an N-terminal (His) ${ }_{6}$-tag and a C-terminal Tag100.

(His) ${ }_{6}$-tagged pro-KLKs were purified from lysed bacteria (in $6 \mathrm{M}$ guanidine hydrochloride, $10 \mathrm{~mm}$ Tris- $\mathrm{HCl}, 100 \mathrm{~mm} \mathrm{NaH}_{2} \mathrm{PO}_{4}, 8 \mathrm{~mm}$ 2-mercaptoethanol, $\mathrm{pH}$ 8.0) via affinity chromatography using a NiNTA Superflow resin (Qiagen) under denaturing conditions. Therefore, proteins were washed and eluted by a $\mathrm{pH}$ gradient: starting off with lysis buffer, the column was washed with $8 \mathrm{M}$ urea, $10 \mathrm{~mm}$ Tris-HCl, $100 \mathrm{~mm} \mathrm{NaH} \mathrm{PO}_{4}, 8$ mм 2-mercaptoethanol, $\mathrm{pH}$ 8.0, 7.0, $6.3,5.9,4.5$, and 4.0, respectively. Most of the recombinant bound pro-KLK was finally eluted with the buffers at $\mathrm{pH} 4.5$ and 4.0. Prior to refolding, the purified pro-KLKs were incubated overnight at room temperature in the elution buffer containing $10 \mathrm{~mm}$ dithiothreitol. For refolding, the urea concentration was subsequently gradually reduced from $8 \mathrm{M}$ to $4 \mathrm{M}, 2 \mathrm{M}$ and $1 \mathrm{M}$ by dialysis. The latter two refolding buffers contained $5 \mathrm{~mm}$ reduced glutathione and $0.5 \mathrm{~mm}$ oxidized glutathione (GSH/GSSG ratio 10:1). Finally, the refolding buffer was exchanged with $100 \mathrm{~mm} \mathrm{NaCl}, 50 \mathrm{~mm}$ Tris$\mathrm{HCl}, \mathrm{pH} 8.0,0.005 \%$ Tween-20, the protein solution cleared by centrifugation at $12000 \mathrm{~g}$, and the purified protein stored at $-20^{\circ} \mathrm{C}$. In addition, using the $\mathrm{pQE}-30$ vector (Qiagen) pro-KLK4 was also expressed and purified as an amino-terminally His-tagged protein.

\section{Exposure of zymogens to potential activators}

Pro-KLKs or pro-uPA, diluted in $100 \mathrm{~mm}$ Tris- $\mathrm{HCl}, 100 \mathrm{~mm} \mathrm{NaCl}$, $\mathrm{pH} 7.5$, were exposed to potential activators, in an enzyme substrate molar ratio of 1:10. In the case of treatment of pro-KLK4 by MMPs, $5 \mathrm{mM} \mathrm{CaCl}_{2}$ was added to the reaction mixture. Activation of proMMP-9 and proMMP-2 was performed by exposure to $1 \mathrm{mM} 4-$ amino-phenylmercuric acetate for $2 \mathrm{~h}$ at $37^{\circ} \mathrm{C}$, and proMMP-9 $\Delta$ was autocatalytically activated by treatment for $1 \mathrm{~h}$ at $37^{\circ} \mathrm{C}$ as described previously (Ries et al., 2007). After incubation of active enzymes with pro-KLKs or pro-uPA for various time periods at $37^{\circ} \mathrm{C}$, the reaction was stopped on ice and samples were prepared for electrophoresis or proteolytic activity measurement (see below).

\section{Protein electrophoresis and immunoblotting}

Proteins were denatured in the presence of $2 \%(\mathrm{w} / \mathrm{v})$ sodium dodecyl sulfate and $5 \%(\mathrm{v} / \mathrm{v}) 2$-mercaptoethanol for $5 \mathrm{~min}$ at $95^{\circ} \mathrm{C}$, followed by SDS-PAGE. Separated proteins were then either Coomassie-stained, silver-stained, or transferred onto polyvinylidene fluoride membranes (Pall, Dreieich, Germany).

For amino-terminal microsequencing, proteins, visualized by Coomassie Blue staining $(0.1 \% \mathrm{w} / \mathrm{v})$ of the membranes, were excised and subjected to automated Edman degradation in a pulse liquid phase sequencer (Procise 492, Applied Biosystems, Foster City, CA, USA).

For (pro-)KLK immunoblots, membranes were blocked with 5\% $(\mathrm{v} / \mathrm{v})$ dried skimmed milk, then probed with an anti-His $\mathrm{mAb}$ (Penta-His, Qiagen, Hilden, Germany; $1 \mu \mathrm{g} / \mathrm{ml}$ ). Bound Abs were thereafter detected by a horseradish peroxidase-conjugated $\mathrm{Ab}$ against mouse Ig (Jackson ImmunoResearch Lab, West Grove, PA, USA; dilution: 1:10 000) followed by a chemiluminescent reaction (ECL, Amersham Biosciences, Little Chalfont, UK). After several washes, membranes were reprobed with an anti-Tag100 mAb (Qiagen; $0.5 \mu \mathrm{g} / \mathrm{ml}$ ).

\section{Proteolytic activity measurement}

For uPA activity measurement, samples were placed in a 96-well plate and adjusted to a $160 \mu \mathrm{l}$ final volume in a $50 \mathrm{~mm}$ Tris- $\mathrm{HCl}$, $150 \mathrm{~mm} \mathrm{NaCl}, \mathrm{pH} 8.0$ buffer, in the presence of $0.13 \mathrm{~mm}$ Pefachrome uPA (Bz- $\beta$-Ala-Gly-Arg-paranitroanilide, Loxo $\mathrm{GmbH}$, Dossenheim, Germany). The release of paranitroanilide at $37^{\circ} \mathrm{C}$ was then monitored at $405 \mathrm{~nm}$ in a spectrophotometer (SLT-Labinstruments, Grödig, Austria) over a 30-min time period and the initial rate of substrate hydrolysis derived from the data was expressed as $\Delta \mathrm{OD}$, in $\mathrm{mU} / \mathrm{min}$

For KLK4 activity measurement, a $50 \mathrm{~mm}$ Tris- $\mathrm{HCl}, 100 \mathrm{~mm}$ $\mathrm{NaCl}, 0.05 \%$ Tween, pH 8.0 buffer, containing $20 \mu \mathrm{M} \mathrm{I-1120} \mathrm{(Boc-}$ Val-Pro-Arg-7-amino-4-methylcoumarin, Bachem, Bubendorf, Switzerland) was used and the release of 7-amino-4-methylcoumarin at $37^{\circ} \mathrm{C}$ was monitored in a spectrofluorimeter (SpectraMax Gemini EM, Molecular Devices, Sunnyvale, CA, USA) over a 30-min time period, with excitation and emission wavelength of 355 and $460 \mathrm{~nm}$, respectively. The initial rate of substrate hydrolysis derived from the data was then expressed as relative fluorescent unit/s.

\section{Acknowledgments}

This study was supported in part by the Kommission Klinische Forschung der TU München, by the Bayerisch-Französisches Hochschulzentrum, by fellowships from the Alexander von Humboldt Stiftung and from the Bayerische Forschungsstiftung (to N.B.), and by an Erasmus student mobility grant (to K.P.). We thank Sandra Baur for her excellent technical assistance, and S. Wagner, M. Valachova and P. Wojciechowski for their help with pro-KLK production.

\section{References}

Barrett, A.J. (2004). Bioinformatics of proteases in the MEROPS database. Curr. Opin. Drug Discov. Dev. 7, 334-341.

Beaufort, N., Debela, M., Creutzburg, S., Kellermann, J., Bode, W., Schmitt, M., Pidard, D., and Magdolen, V. (2006). Interplay of human tissue kallikrein 4 (hK4) with the plasminogen activation system: hK4 regulates the structure and functions of the urokinase-type plasminogen activator receptor (UPAR). Biol. Chem. 387, 217-222.

Becker-Pauly, C., Howel, M., Walker, T., Vlad, A., Aufenvenne, K., Oji, V., Lottaz, D., Sterchi, E.E., Debela, M., Magdolen, V., et al. (2007). The $\alpha$ and $\beta$ subunits of the metalloprotease meprin are expressed in separate layers of human epidermis, revealing different functions in keratinocyte proliferation and differentiation. J. Invest. Dermatol. 127, 1115-1125.

Borgono, C.A. and Diamandis, E.P. (2004). The emerging roles of human tissue kallikreins in cancer. Nat. Rev. Cancer 4, 876-890.

Dano, K., Behrendt, N., Hoyer-Hansen, G., Johnsen, M., Lund, L.R., Ploug, M., and Romer, J. (2005). Plasminogen activation and cancer. Thromb. Haemost. 93, 676-681.

Debela, M., Magdolen, V., Grimminger, V., Sommerhoff, C., Messerschmidt, A., Huber, R., Friedrich, R., Bode, W., and Goettig, P. (2006a). Crystal structures of human tissue kallikrein 4: activity modulation by a specific zinc binding site. J. Mol. Biol. 362, 1094-1107.

Debela, M., Magdolen, V., Schechter, N., Valachova, M., Lottspeich, F., Craik, C.S., Choe, Y., Bode, W., and Goettig, P. (2006b). Specificity profiling of seven human tissue kallikreins reveals individual subsite preferences. J. Biol. Chem. 281, 25678-25688. 
Debela, M., Goettig, P., Magdolen, V., Huber, R., Schechter, N.M., and Bode, W. (2007). Structural basis of the zinc inhibition of human tissue kallikrein 5. J. Mol. Biol. 373, 1017-1031.

Debela, M., Beaufort, N., Magdolen, V., Schechter, N.M., Craik, C.S., Schmitt, M., Bode, W., and Goettig, P. (2008). Structures and specificity of the human kallikrein-related peptidases KLK 4, 5, 6, and 7. Biol. Chem. 389, 623-632.

Emami, N. and Diamandis, E.P. (2010). Potential role of multiple members of the kallikrein-related peptidase family of serine proteases in activating latent $\mathrm{TGFb} 1$ in semen. Biol. Chem. 391, $85-95$.

Freije, J.M., Balbin, M., Pendas, A.M., Sanchez, L.M., Puente, X.S., and Lopez-Otin, C. (2003). Matrix metalloproteinases and tumor progression. Adv. Exp. Med. Biol. 532, 91-107.

Frenette, G., Tremblay, R.R., Lazure, C., and Dube, J.Y. (1997). Prostatic kallikrein hK2, but not prostate-specific antigen (hK3), activates single-chain urokinase-type plasminogen activator. Int. J. Cancer 71, 897-899.

Gomis-Rüth, F.X., Bayés, A., Sotiropoulou, G., Pampalakis, G., Tsetsenis, T., Villegas, V., Avilés, F.X., and Coll, M. (2002). The structure of human prokallikrein 6 reveals a novel activation mechanism for the kallikrein family. J. Biol. Chem. 277, 27273-27281.

Hollenberg, M.D., Oikonomopoulou, K., Hansen, K.K., Saifeddine, M., Ramachandran, R., and Diamandis, E.P. (2008). Kallikreins and proteinase-mediated signaling: proteinase-activated receptors (PARs) and the pathophysiology of inflammatory diseases and cancer. Biol. Chem. 389, 643-651.

Itoh, Y. and Nagase, H. (2002). Matrix metalloproteinases in cancer. Essays Biochem. 38, 21-36.

Jung, K., Nowak, L., Lein, M., Priem, F., Schnorr, D., and Loening, S.A. (1997). Matrix metalloproteinases 1 and 3, tissue inhibitor of metalloproteinase-1 and the complex of metalloproteinase-1/ tissue inhibitor in plasma of patients with prostate cancer. Int. J. Cancer 74, 220-223.

Lopez-Otin, C. and Bond, J.S. (2008). Proteases: multifunctional enzymes in life and disease. J. Biol. Chem. 283, 30433-30437.

Ohler, A., Debela, M., Wagner, S., Magdolen, V., and Becker-Pauly, C. (2010). Analyzing the protease web in skin: meprin metalloproteases are activated specifically by KLK4, 5 and 8 vice versa leading to processing of proKLK7 thereby triggering its activation. Biol. Chem. 391, 455-460.

Pampalakis, G. and Sotiropoulou, G. (2007). Tissue kallikrein proteolytic cascade pathways in normal physiology and cancer. Biochim. Biophys. Acta 1776, 22-31.

Ramsay, A.J., Dong, Y., Hunt, M.L., Linn, M., Samaratunga, H., Clements, J.A., and Hooper, J.D. (2008). Kallikrein-related peptidase 4 (KLK4) initiates intracellular signaling via protease-acti- vated receptors (PARs). KLK4 and PAR-2 are co-expressed during prostate cancer progression. J. Biol. Chem. 283, $12293-$ 12304.

Ries, C., Pitsch, T., Mentele, R., Zahler, S., Egea, V., Nagase, H., and Jochum, M. (2007). Identification of a novel $82 \mathrm{kDa}$ proMMP-9 species associated with the surface of leukaemic cells: (auto-)catalytic activation and resistance to inhibition by TIMP-1. Biochem. J. 405, 547-558.

Rittenhouse, H.G., Finlay, J.A., Mikolajczyk, S.D., and Partin, A.W. (1998). Human kallikrein 2 (hK2) and prostate-specific antigen (PSA): two closely related, but distinct, kallikreins in the prostate. Crit. Rev. Clin. Lab. Sci. 35, 275-368.

Ryu, O., Hu, J.C., Yamakoshi, Y., Villemain, J.L., Cao, X., Zhang, C., Bartlett, J.D., and Simmer, J.P. (2002). Porcine kallikrein-4 activation, glycosylation, activity, and expression in prokaryotic and eukaryotic hosts. Eur. J. Oral Sci. 110, 358-365.

Shaw, J.L. and Diamandis, E.P. (2007). Distribution of 15 human kallikreins in tissues and biological fluids. Clin. Chem. 53, 1423-1432.

Sperl, S., Mueller, M.M., Wilhelm, O.G., Schmitt, M., Magdolen, V., and Moroder, L. (2001). The uPA/uPA receptor system as a target for tumor therapy. Drug News Perspect. 14, 401-411.

Takayama, T.K., Fujikawa, K., and Davie, E.W. (1997). Characterization of the precursor of prostate-specific antigen. Activation by trypsin and by human glandular kallikrein. J. Biol. Chem. 272, 21582-21588.

Takayama, T.K., McMullen, B.A., Nelson, P.S., Matsumura, M., and Fujikawa, K. (2001). Characterization of hK4 (prostase), a prostate-specific serine protease: activation of the precursor of prostate specific antigen (pro-PSA) and single-chain urokinase-type plasminogen activator and degradation of prostatic acid phosphatase. Biochemistry 40, 15341-15348.

Tye, C.E., Pham, C.T., Simmer, J.P., and Bartlett, J.D. (2009). DPPI may activate KLK4 during enamel formation. J. Dent. Res. 88, 323-327.

Yoon, H., Laxmikanthan, G., Lee, J., Blaber, S.I., Rodriguez, A., Kogot, J.M., Scarisbrick, I.A., and Blaber, M. (2007). Activation profiles and regulatory cascades of the human kallikrein-related peptidases. J. Biol. Chem. 282, 31852-31864.

Yoon, H., Blaber, S.I., Evans, D.M., Trim, J., Juliano, M.A., Scarisbrick, I.A., and Blaber, M. (2008). Activation profiles of human kallikrein-related peptidases by proteases of the thrombostasis axis. Protein Sci. 17, 1998-2007.

Yoon, H., Blaber, S.I., Debela, M., Goettig, P., Scarisbrick, I.A., and Blaber, M. (2009). A completed KLK activome profile: investigation of activation profiles of KLK9, 10, and 15. Biol. Chem. 390, 373-377.

Received January 5, 2010; accepted February 9, 2010 